Journal of Organometallic Chemistry, 410 (1991) C13-C19

Elsevier Sequoia S.A., Lausanne

JOM 21883PC

Preliminary communication

\title{
Tetra-tert-butyl-pentafulvalen als Ligand in zweikernigen Molybdänkomplexen: cis-Anordnung ohne Metall-Metall-Bindung
}

\author{
Peter Jutzi *, Jörg Schnittger, Beate Neumann und Hans-Georg Stammler \\ Fakultät für Chemie der Universität Bielefeld, Universitätsstraße 25, W-4800 Bielefeld 1 (Deutschland) \\ (Eingegangen den 21. März 1991)
}

\begin{abstract}
Reaction of 1,1',3,3'-tetra-tert-butyl-5-5'-pentafulvalenedipotassium (1) with hexacarbonylmolybdenum leads to hexacarbonyl-dipotassium $\left(1,1^{\prime}, 3,3^{\prime}\right.$-tetra-tert-butyl-5, $5^{\prime}$-pentafulvalene)dimolybdate (2), which on further treatment with stoichiometric amounts of iodomethane yields the hexacarbonyldimethyl(1,1',3,3'-tetra-tert-butyl-5,5'-pentafulvalene)dimolybdenum, $\left(\eta^{5}: \eta^{5}-{ }^{\prime} \mathrm{Bu}_{4} \mathrm{C}_{10} \mathrm{H}_{4}\right) \mathrm{Mo}_{2}(\mathrm{CO})_{6}$ $\left(\mathrm{CH}_{3}\right)_{2}$ (3). Compound 3 is obtained as yellow needles and brownish cube-like crystals, and it is characterized by ${ }^{1} \mathrm{H}-\mathrm{NMR},{ }^{13} \mathrm{C}-\mathrm{NMR}$, IR, and MS data. The cubes crystallize in the space group $\mathrm{C} 2 / \mathrm{c}$ with four molecules in the unit cell. Each molecule consists of two tricarbonylmethyl(cyclopentadienyl) molybdenum units which are connected by a central C-C-bond, twisted against each other by $64.8^{\circ}$ and bent by $25.8^{\circ}$. Due to the steric requirements of the tert-butyl substituents in the fulvalene ligand, 3 should be formed only from cis-configurated 2.
\end{abstract}

Vor kurzem berichteten wir über die Synthese und die Festkörperstruktur von 1,1',3,3'-Tetra-tert-butyl-5,5'-pentafulvalen-dithallium [1]. Während in Pentafulvalen-Komplexen ohne Metall-Metall-Bindung eine trans-Anordnung der ZentralAtome typisch ist [2-4], wird bei der von uns dargestellten Dithallium-Verbindung erstmals eine cisoide Konfiguration gefunden, obwohl keine intramolekulare Tl-TlWechselwirkung vorliegt.

Im Rahmen unserer experimentellen Arbeiten mit dem $\pi$-Ligandensystem Tetratert-butyl-pentafulvalen war für uns daher die Frage von Bedeutung, ob die beobachtete cis-Anordnung ein Einzelfall ist, oder ob sich weitere Beispiele finden lassen, in welchen nicht über Metall-Metall-Bindung verknüpfte Zentral-Atome cis-ständig orientiert sind. Besonderes Interesse galt in diesem Zusammenhang Komplexen aus der Übergangsmetall-Chemie. Eine cis-Anordnung von Metallzentren sollte zum Studium von cooperativen Effekten interessant sein.

Wir berichten im Folgenden über die Darstellung von 1,1', $3,3^{\prime}$-Tetra-tert-butyl5,5'-pentafulvalen-dikalium (1), $\left(\eta^{5}: \eta^{5}-{ }^{t} \mathrm{Bu}_{4} \mathrm{C}_{10} \mathrm{H}_{4}\right) \mathrm{K}_{2}$, Hexacarbonyl-dikalium(1,$1^{\prime}, 3,3^{\prime}$-tetra-tert-butyl-5,5'-pentafulvalen)dimolybdat (2), $\left(\eta^{5}: \eta^{5}-{ }^{1} \mathrm{Bu}_{4} \mathrm{C}_{10} \mathrm{H}_{4}\right) \mathrm{Mo}_{2}$ (CO) ${ }_{6} \mathrm{~K}_{2}$, und Hexacarbonyl-dimethyl $\left(1,1^{\prime}, 3,3^{\prime}\right.$-tetra-tert-butyl-5,5'-pentafulvalen)dimolybdän, $\left(\eta^{5}: \eta^{5}-{ }^{t} \mathrm{Bu}_{4} \mathrm{C}_{10} \mathrm{H}_{4}\right) \mathrm{Mo}_{2}(\mathrm{CO})_{6}\left(\mathrm{CH}_{3}\right)_{2}$ (3). Die Verbindungen 2 und 3 
sind die ersten Übergangsmetall-Komplexe auf Pentafulvalen-Basis ohne MetallMetall-Bindung mit cisoider Struktur.

\section{Präparative Ergebnisse}

Setzt man eine THF-Lösung von 1,1',3,3'-Tetra-tert-butyl-5,5'-dihydropentafulvalen [5] mit stöchiometrischen Mengen Kaliumhydrid um, so bildet sich unter Wasserstoff-Entwicklung bei Raumtemperatur 1,1',3,3'-Tetra-tert-butyl-5,5'-pentafulvalen-dikalium (1). Verbindung 1 fällt quantitativ als hellgelbes amorphes Pulver aus. Nach Entfernen des Tetrahydrofurans wird 1 in Anlehnung an bekannte Reaktionsprinzipien [6-8] mit Hexacarbonylmolybdän in siedendem DMF umgesetzt. Das unter CO-Entwicklung gebildete Hexacarbonyl-dikalium(1,1',3,3'-tetratert-butyl-5,5'-pentafulvalen)dimolybdat (2) wird durch Abdestillieren des Solvens isoliert. Anschließend wird eine THF-Lösung von 2 mit stöchiometrischen Mengen Iodmethan versetzt, wobei sich spontan ein Niederschlag von Kaliumiodid bildet. Nach der üblichen Aufarbeitung der Reaktionslösung erhält man Hexacarbonyl-dimethyl(1,1',3,3'-tetra-tert-butyl-5,5'-pentafulvalen)dimolybdän, ( $\eta^{5}: \eta^{5}-{ }^{t} \mathrm{Bu}_{4^{-}}$ $\left.\mathrm{C}_{10} \mathrm{H}_{4}\right) \mathrm{Mo}_{2}(\mathrm{CO})_{6}\left(\mathrm{CH}_{3}\right)_{2}(3)$, in befriedigender Ausbeute.

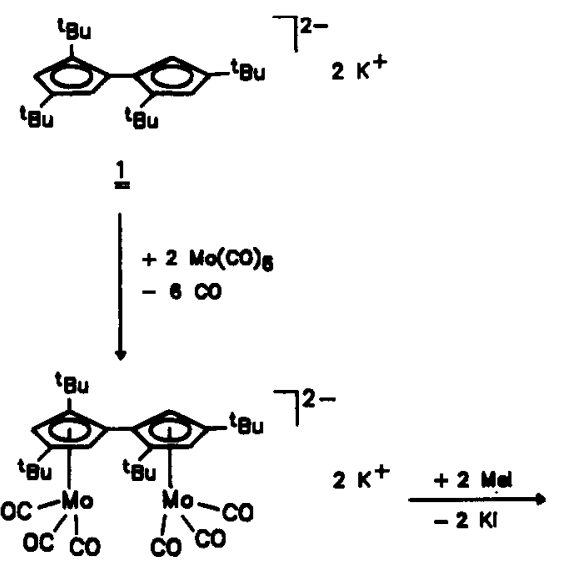

$\underline{2}$

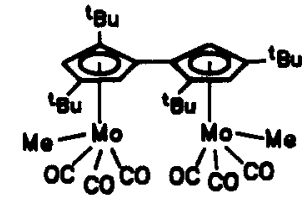

3

Verbindung 3 kristallisiert aus Petrolether in zwei unterschiedlichen Modifikationen: Es werden sowohl goldgelbe, würfelförmige Kristalle als auch blaßgelbe Nadeln isoliert. Durch Umkristallisieren aus Dichlormethan-Petrolether ist es möglich, die Würfel in Nadeln zu überführen. Sowohl die Würfel als auch die Nadeln schmelzen unter Zersetzung bei $181^{\circ} \mathrm{C}$.

Die Kristallmodifikationen ergeben identische NMR-Spektren. Man erkennt im ${ }^{1}$ H-NMR-Spektrum zwei Signale für die magnetisch unterschiedlichen tert-ButylEinheiten am Fulvalengerüst sowie zwei Resonanzen für die am Fünfring gebundenen Protonen; letztere haben Dublett-Charakter, die Kopplungskonstante beträgt $2.6 \mathrm{~Hz}$. Das Signal für die an den Molybdän-Kernen gebundenen MethylGruppen erscheint bei $0.80 \mathrm{ppm}\left(\mathrm{C}_{6} \mathrm{D}_{6}\right)$. Im ${ }^{13} \mathrm{C}-\mathrm{NMR}-\mathrm{Spektrum}\left(\mathrm{C}_{6} \mathrm{D}_{6}\right)$ beobachtet man neben den erwarteten vier Signalen für die tert-Butyl-Einheiten fünf Reso- 


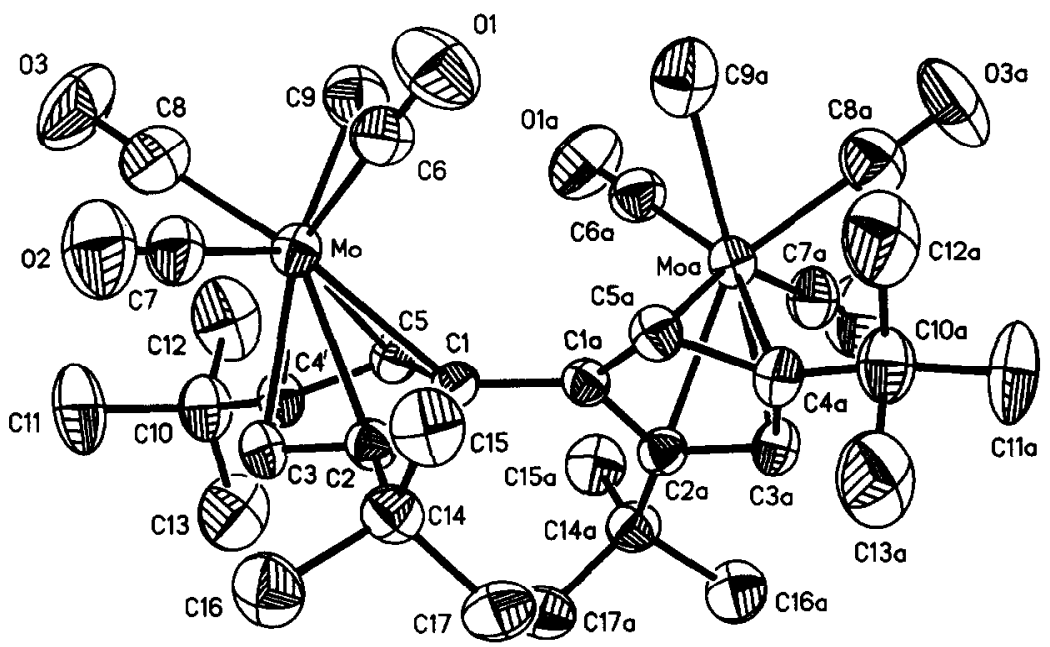

Fig. 1. Molekülstruktur von 3.

nanzen für die Ringkohlenstoff-Atome im Bereich von 93.05-124.29 ppm. Die ${ }^{13} \mathrm{C}-$ Resonanz für die metallgebundenen Methyl-Gruppen liegt bei $-17.96 \mathrm{ppm}$. Es werden drei Signale für die jeweils chemisch und magnetisch unterschiedlichen Carbonyl-Einheiten beobachtet.

Beide Modifikationen liefern nahezu identische Infrarot-Spektren. Im Bereich der CO-Valenzschwingungen erkennt man jeweils drei Banden, die auf terminale COGruppen hinweisen. Im Falle der würfelförmigen Modifikation liegen sie bei 1912, 1931 und $2006 \mathrm{~cm}^{-1}$, im Falle der nadeligen Modifikation bei 1906, 1926 und 2015 $\mathrm{cm}^{-1}$. Beide Modifikationen ergeben identische Massenspektren: man beobachtet das Molekül-Ion bei $m / z=742$; der Basispeak ist dem Fragment-Ion $m / z=28$ $\left(\mathrm{CO}^{+}\right)$zuzuordnen.

Da die beschriebenen Daten keine Aussagen über die Konfiguration des Komplexes 3 gestatten, wurde eine Kristallstrukturanalyse durchgeführt.

\section{Kristallstruktur von 3}

Die Röntgenstrukturanalyse [9*] der würfelförmigen Modifikation von 3 zeigt zwei isolierte Tricarbonyl-methyl(ditert-butyl-cyclopentadienyl)molybdän-Einheiten über eine $\mathrm{C}_{0}$-Brücke [C(1)-C(1a)] miteinander verbunden (Fig. 1). Beide Fragmente sind in bezug auf diese Achse um den Betrag von $64.8^{\circ}$ gegeneinander verdreht und zusätzlich um $25.8^{\circ}$ abgewinkelt. Aus dieser Stellung resultiert zwangsläufig eine cisoide Konstellation der Metall-Atome [1] (Fig. 2). Die tert-Butyl-Gruppen beider Cp-Fragmente sind durch diese Drehung deutlich, wenn auch nicht maximal, voneinander entfernt. Die zu 1.483(9) $\AA$ bestimmte Distanz für die C(1)-C(1a)-Bindung entspricht einer Einfachbindung und ist für Komplexe des Pentafulvalens nicht ungewöhnlich [1-3,10,11].

\footnotetext{
* Die Literaturnummer mit einem Sternchen deutet eine Bemerkung in der Literaturliste an.
} 


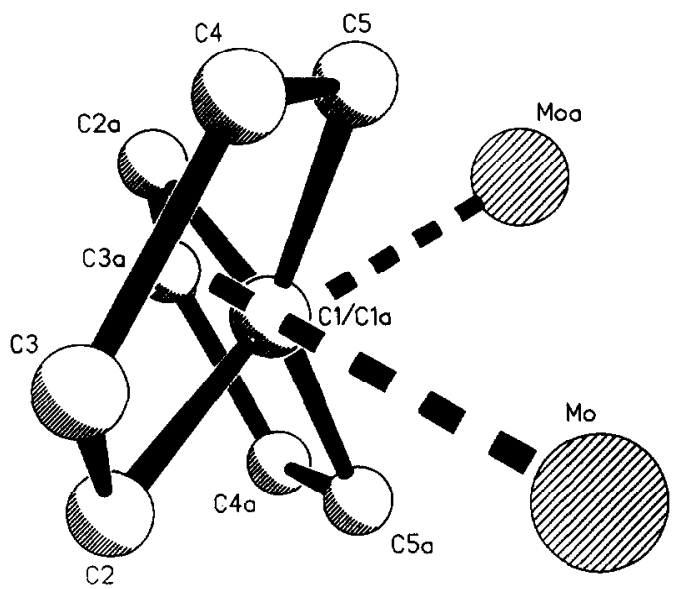

Fig. 2. Seitenansicht von 3.

Die basalen Fünfringe sind zu den Molybdän-Atomen nicht exakt pentahapto koordiniert. Die Abstände des Metalls zu den Kohlenstoff-Atomen des Cp-Gerüstes schwanken zwischen 2.315(5) $\AA$ [Mo-C(3)] und 2.396(4) $\AA$ [Mo-C(1)]. Durch Mittelung dieser Werte resultiert die Distanz zum Mittelpunkt des Cp-Ringes zu $2.025 \AA$ Å. Die C-C-Abstände im aromatischen Fulvalen-Grundgerüst unterscheiden sich wenig: sie differieren zwischen 1.413 (7) $\AA$ [C(3)-C(4)] und 1.448 (5) $\AA$ $[C(1)-C(2)]$.

Die C-Atome der restlichen drei CO-Liganden und der Methyl-Gruppe bilden in dieser Anordnung eine verzerrte quadratische Pyramide mit dem Mo-Atom als Spitze. Die Winkel $\mathrm{C}(\mathrm{O})-\mathrm{Mo}-\mathrm{C}(\mathrm{H})$ bzw. $\mathrm{C}(\mathrm{O})-\mathrm{Mo}-\mathrm{C}(\mathrm{O})$ schwanken zwischen 70.1(2) ${ }^{\circ}[\mathrm{C}(8)-\mathrm{Mo}-\mathrm{C}(9)]$ und $78.7(2)^{\circ}[\mathrm{C}(6)-\mathrm{Mo}-\mathrm{C}(7)]$. Im Einklang mit strukturell vergleichbaren Tricarbonyl(cyclopentadienyl)molybdän-Einheiten liegen die Mo$\mathrm{C}(\mathrm{O})$ Distanzen mit 1.972(7) $\AA$ [Mo-C(6)] bis 1.994(5) $\AA$ [Mo-C(7)] im üblichen Bereich $[12,13]$. Die CO Bindungslängen liegen im Bereich von 1.130(6) $\AA$ [C(7)-

Tabelle 1

Bindungslängen $(\AA)$ in 3 [9]

\begin{tabular}{llll}
\hline Mo-C(1) & $2.396(4)$ & $M o-C(2)$ & $2.363(5)$ \\
Mo-C(3) & $2.315(5)$ & $M o-C(4)$ & $2.375(6)$ \\
Mo-C(5) & $2.357(4)$ & $M o-C(6)$ & $1.972(7)$ \\
Mo-C(7) & $1.994(5)$ & $M o-C(8)$ & $1.984(7)$ \\
Mo-C(9) & $2.347(5)$ & $O(1)-C(6)$ & $1.145(9)$ \\
$O(2)-C(7)$ & $1.130(6)$ & $O(3)-C(8)$ & $1.145(9)$ \\
$C(1)-C(2)$ & $1.448(5)$ & $C(1)-C(5)$ & $1.427(9)$ \\
$C(1)-C(1 A)$ & $1.483(9)$ & $C(2)-C(3)$ & $1.436(9)$ \\
$C(2)-C(14)$ & $1.545(8)$ & $C(3)-C(4)$ & $1.413(7)$ \\
$C(4)-C(5)$ & $1.420(6)$ & $C(4)-C(10)$ & $1.521(10)$ \\
$C(10)-C(11)$ & $1.541(6)$ & $C(10)-C(12)$ & $1.506(9)$ \\
$C(10)-C(13)$ & $1.510(9)$ & $C(14)-C(15)$ & $1.537(9)$ \\
$C(14)-C(16)$ & $1.541(7)$ & $C(14)-C(17)$ & $1.532(7)$ \\
\hline
\end{tabular}


Tabelle 2

Bindungswinkel $\left(^{\circ}\right)$ in 3

\begin{tabular}{|c|c|c|c|}
\hline $\mathrm{C}(1)-\mathrm{Mo}-\mathrm{C}(2)$ & $35.4(1)$ & $\mathrm{C}(1)-\mathrm{Mo}-\mathrm{C}(3)$ & $58.3(1)$ \\
\hline $\mathrm{C}(2)-\mathrm{Mo}-\mathrm{C}(3)$ & $35.7(2)$ & $\mathrm{C}(1)-\mathrm{Mo}-\mathrm{C}(4)$ & $58.6(2)$ \\
\hline $\mathrm{C}(2)-\mathrm{Mo}-\mathrm{C}(4)$ & $59.5(2)$ & $\mathrm{C}(3)-\mathrm{Mo}-\mathrm{C}(4)$ & $35.0(2)$ \\
\hline $\mathrm{C}(1)-\mathrm{Mo}-\mathrm{C}(5)$ & $34.9(2)$ & $\mathrm{C}(2)-\mathrm{Mo}-\mathrm{C}(5)$ & $58.8(2)$ \\
\hline $\mathrm{C}(3)-\mathrm{Mo}-\mathrm{C}(5)$ & $57.8(1)$ & $\mathrm{C}(4)-\mathrm{Mo}-\mathrm{C}(5)$ & $34.9(2)$ \\
\hline $\mathrm{C}(1)-\mathrm{Mo}-\mathrm{C}(6)$ & $98.1(2)$ & $\mathrm{C}(2)-\mathrm{Mo}-\mathrm{C}(6)$ & $105.2(2)$ \\
\hline $\mathrm{C}(3)-\mathrm{Mo}-\mathrm{C}(6)$ & $139.0(2)$ & $\mathrm{C}(4)-\mathrm{Mo}-\mathrm{C}(6)$ & $156.2(2)$ \\
\hline$C(5)-M o-C(6)$ & $122.5(2)$ & $\mathrm{C}(1)-\mathrm{Mo}-\mathrm{C}(7)$ & $125.9(2)$ \\
\hline $\mathrm{C}(2)-\mathrm{Mo}-\mathrm{C}(7)$ & $92.7(2)$ & $\mathrm{C}(3)-\mathrm{Mo}-\mathrm{C}(7)$ & $89.2(2)$ \\
\hline $\mathrm{C}(4)-\mathrm{Mo}-\mathrm{C}(7)$ & $117.9(2)$ & $\mathrm{C}(5)-\mathrm{Mo}-\mathrm{C}(7)$ & $146.7(2)$ \\
\hline $\mathrm{C}(6)-\mathrm{Mo}-\mathrm{C}(7)$ & $78.7(2)$ & $\mathrm{C}(1)-\mathrm{Mo}-\mathrm{C}(8)$ & 152.1(3) \\
\hline $\mathrm{C}(2)-\mathrm{Mo}-\mathrm{C}(8)$ & $145.2(2)$ & $\mathrm{C}(3)-\mathrm{Mo}-\mathrm{C}(8)$ & $110.3(2)$ \\
\hline$C(4)-M o-C(8)$ & $96.9(3)$ & $\mathrm{C}(5)-\mathrm{Mo}-\mathrm{C}(8)$ & $117.2(3)$ \\
\hline $\mathrm{C}(6)-\mathrm{Mo}-\mathrm{C}(8)$ & $104.3(3)$ & $\mathrm{C}(7)-\mathrm{Mo}-\mathrm{C}(8)$ & $75.5(2)$ \\
\hline$C(1)-M o-C(9)$ & $100.0(2)$ & $C(2)-M o-C(9)$ & $135.4(1)$ \\
\hline $\mathrm{C}(3)-\mathrm{Mo}-\mathrm{C}(9)$ & $136.2(2)$ & $\mathrm{C}(4)-\mathrm{Mo}-\mathrm{C}(9)$ & $101.5(2)$ \\
\hline $\mathrm{C}(5)-\mathrm{Mo}-\mathrm{C}(9)$ & $82.5(2)$ & $\mathrm{C}(6)-\mathrm{Mo}-\mathrm{C}(9)$ & $76.1(3)$ \\
\hline $\mathrm{C}(7)-\mathrm{Mo}-\mathrm{C}(9)$ & $130.0(2)$ & $\mathrm{C}(8)-\mathrm{Mo}-\mathrm{C}(9)$ & $70.1(2)$ \\
\hline $\mathrm{Mo}-\mathrm{C}(1)-\mathrm{C}(2)$ & $71.1(2)$ & $\mathrm{Mo}-\mathrm{C}(1)-\mathrm{C}(5)$ & $71.0(2)$ \\
\hline$C(2)-C(1)-C(5)$ & $107.3(4)$ & $M o-C(1)-C(1 A)$ & $135.2(3)$ \\
\hline$C(2)-C(1)-C(1 A)$ & $125.9(6)$ & $C(5)-C(1)-C(1 A)$ & $125.0(5)$ \\
\hline $\mathrm{Mo}-\mathrm{C}(2)-\mathrm{C}(1)$ & $73.5(2)$ & $\mathrm{Mo}-\mathrm{C}(2)-\mathrm{C}(3)$ & $70.3(3)$ \\
\hline$C(1)-C(2)-C(3)$ & $105.5(5)$ & Mo-C(2)-C(14) & $127.8(3)$ \\
\hline$C(1)-C(2)-C(14)$ & $130.0(5)$ & $C(3)-C(2)-C(14)$ & $123.9(4)$ \\
\hline $\mathrm{Mo}-\mathrm{C}(3)-\mathrm{C}(2)$ & $74.0(3)$ & Mo-C(3)-C(4) & $74.8(3)$ \\
\hline$C(2)-C(3)-C(4)$ & $111.2(4)$ & $\mathrm{Mo}-\mathrm{C}(4)-\mathrm{C}(3)$ & $70.2(3)$ \\
\hline $\mathrm{Mo}-\mathrm{C}(4)-\mathrm{C}(5)$ & $71.8(3)$ & $C(3)-C(4)-C(5)$ & $105.6(5)$ \\
\hline$M o-C(4)-C(10)$ & $130.8(3)$ & $C(3)-C(4)-C(10)$ & $127.6(4)$ \\
\hline$C(5)-C(4)-C(10)$ & $125.9(4)$ & Mo-C(5)-C(1) & $74.0(2)$ \\
\hline Mo-C(5)-C(4) & $73.3(2)$ & $C(1)-C(5)-C(4)$ & $110.3(4)$ \\
\hline Mo-C(6)-O(1) & $177.9(5)$ & Mo-C(7)-O(2) & $178.7(5)$ \\
\hline Mo-C(8)-O(3) & $179.4(6)$ & $C(4)-C(10)-C(11)$ & $111.3(5)$ \\
\hline$C(4)-C(10)-C(12)$ & $111.8(5)$ & $C(11)-C(10)-C(12)$ & $108.7(5)$ \\
\hline$C(4)-C(10)-C(13)$ & $106.9(5)$ & $C(11)-C(10)-C(13)$ & $108.9(5)$ \\
\hline$C(12)-C(10)-C(13)$ & $109.2(5)$ & $C(2)-C(14)-C(15)$ & $114.8(4)$ \\
\hline$C(2)-C(14)-C(16)$ & $109.8(5)$ & $C(15)-C(14)-C(16)$ & $106.7(4)$ \\
\hline$C(2)-C(14)-C(17)$ & $109.4(4)$ & $C(15)-C(14)-C(17)$ & $108.4(6)$ \\
\hline$C(16)-C(14)-C(17)$ & $107.5(4)$ & & \\
\hline
\end{tabular}

$\mathrm{O}(2)]$ bis $1.145(9) \AA ̊(C(6)-O(1), C(8)-O(3)]$. Die Distanz Mo-C(H) besträgt 2.347(5) $\AA$.

\section{Fazit}

Hexacarbonyl-dimethyl(1,1',3,3'-tetra-tert-butyl-5,5'-pentafulvalen)dimolybdän, $\left(\eta^{5}: \eta^{5}-{ }^{-} \mathrm{Bu}_{4} \mathrm{C}_{10} \mathrm{H}_{4}\right) \mathrm{Mo}_{2}(\mathrm{CO})_{6}\left(\mathrm{CH}_{3}\right)_{2}(3)$, ist das erste Beispiel eines Übergangsmetall-Komplexes auf Pentafulvalen-Basis ohne Metall-Metall-Bindung mit cisoider Anordnung der Metall-Atome. Trotz der raumerfüllenden Liganden an den Molybdän-Zentren (je drei CO-Gruppen und eine $\mathrm{CH}_{3}$-Einheit) sind keine Hinweise auf die sterisch günstigere trans-Konfiguration vorhanden. Vermutlich liegt bereits 
in 1,1',3,3'-Tetra-tert-butyl-5,5'-pentafulvalen-dikalium (1) eine cis-Anordnung vor, die während der gesamten Reaktionssequenz beibehalten wird. Auch im Dikaliumhexacarbonyl(1,1',3,3'-tetra-tert-butyl-5,5'-pentafulvalen)dimolybdat (2) wäre eine trans-Anordnung aus elektrostatischen Gründen energetisch günstiger. Eine Rotation zur Ausbildung einer transoiden Konfiguration, wie sie für viele FulvalenKomplexe ohne Metall-Metall-Bindung typisch ist [2-4], ist jedoch durch die raumerfüllenden tert-Butyl-Gruppen in 1- und 1'-Stellung gehindert. Somit bietet der Tetra-tert-butyl-pentafulvalen-Ligand günstige Voraussetzungen zur Synthese von Zweikern-Komplexen mit cis-Anordnung von Metallzentren, die nicht direkt miteinander verbunden sind.

\section{Experimenteller Teil}

Sämtliche Arbeiten wurden unter Ausschluß von Luft und Feuchtigkeit untder Argon durchgeführt; verwendete Geräte, Chemikalien und Lösungsmittel waren entsprechend vorbereitet. Schmelzpunkt (n. korr.): Büchi 510 Schmelzpunktbestimmungsapparatur, Probenröhrchen abgeschmolzen. NMR (Bruker AM 300): ${ }^{\mathbf{l}} \mathbf{H}-$ NMR (300.1 MHz) ext. TMS, ${ }^{13} \mathrm{C}\left\{{ }^{1} \mathrm{H}\right\}$-NMR (75.4 MHz) ext. TMS. Massenspektrum: Finnigan MAT 311 A (70 eV, $300 \mu$ A Emission); es sind nur charakteristische Fragment-Ionen angegeben. $\mathrm{CH}$-Analyse: Mikroanalytisches Laboratorium Beller, Göttingen. IR-Spektren: Mattson Polaris FTIR/Atari 1040 ST.

$\left(1,1^{\prime}, 3,3^{\prime}\right.$-Tetra-tert-butyl-5,5'-pentafulvalen)dikalium (1). $0.65 \mathrm{~g}$ (16.2 mmol) Kaliumhydrid werden in $30 \mathrm{ml}$ Tetrahydrofuran suspendiert und unter Eiskühlung mit einer Lösung von $2.85 \mathrm{~g}$ (8.10 mmol) $1,1^{\prime}, 3,3^{\prime}$-Tetra-tert-butyl-5,5'-dihydropentafulvalen in $20 \mathrm{ml}$ THF versetzt. Man erwärmt auf Raumtemperatur, wobei Gasentwicklung einsetzt und 1 als hellgelber, amorpher Niederschlag ausfällt; 1 wird ohne weitere Reinigung eingesetzt.

Hexacarbonyl-dikalium(1,1',3,3'-tetra-tert-butyl-5,5'-pentafulvalen)dimolybdat (2). $\mathrm{Zu}$ einer Suspension von $8.10 \mathrm{mmol} 1$ in $40 \mathrm{ml}$ DMF gibt man $4.30 \mathrm{~g}$ (16.3 mmol) Hexacarbonylmolybdän. Man erwärmt $3 \mathrm{~h}$ auf $130^{\circ} \mathrm{C}$, wobei heftige Gasentwicklung einsetzt. Die nun gelb-braune Lösung wird noch weitere $12 \mathrm{~h}$ bei Raumtemperatur gerührt. Man entfernt das Solvens i. Vak.. 2 wird ohne Reinigung eingesetzt.

Hexacarbonyl-dimethyl(1,1',3,3'-tetra-tert-butyl-5,5'-pentafulvalen)dimolybdän (3). Eine Lösung von $8.10 \mathrm{mmol} 2$ in $50 \mathrm{ml}$ THF wird auf $0^{\circ} \mathrm{C}$ abgekühlt und mit $2.30 \mathrm{~g}$ (16.3 mmol) Iodmethan versetzt, wobei sich sofort ein hellbrauner Niederschlag bildet. Man erwärmt $3 \mathrm{~h}$ auf $50^{\circ} \mathrm{C}$, destilliert das Solvens i. Vak. ab, nimmt den Rückstand in $80 \mathrm{ml}$ Diethylether auf und filtriert. Das Filtrat wird i. Vak. eingeengt und auf $0^{\circ} \mathrm{C}$ abgekühlt, wobei 3 in Form von braunen Würfeln und gelben Nadeln isoliert wird. Umkristallisation der Würfel aus Dichlormethan/Petrolether liefert gelbe Nadeln. Ausbeute: $1.35 \mathrm{~g}(22 \%)$, Schmp.: $181^{\circ} \mathrm{C}$.

${ }^{1} \mathrm{H}-\mathrm{NMR}\left(\mathrm{C}_{6} \mathrm{D}_{6}\right): \delta=0.80(\mathrm{~s}, 6 \mathrm{H}, 2 \times \mathrm{Me}), 1.04,1.05\left(2 \mathrm{~s}, 2 \times 18 \mathrm{H}, 2 \times 2{ }^{\mathrm{t}} \mathrm{Bu}\right)$, $4.88,5.25\left(2 \mathrm{~d},{ }^{4} \mathrm{~J}(\mathrm{HH})=2.6 \mathrm{~Hz}, 2 \times 2 \mathrm{Cp}-\mathrm{H}\right) .{ }^{13} \mathrm{C}-\mathrm{NMR}\left(\mathrm{C}_{6} \mathrm{D}_{6}\right): \delta=-17.96(\mathrm{Me})$, 31.34, $33.73\left(\mathrm{CMe}_{3}\right), 31.96,33.50\left(\mathrm{CMe}_{3}\right)$, 93.05, 99.96 (C-1,3-Ring), 106.82 (C-5Ring), 123.87, 124.29 (C-2,4-Ring), 228.09, 228.57, 241.36 (CO). IR (CsI-Preßling): Würfel: $\nu=1912,1931,2006 \mathrm{~cm}^{-1}$ (CO); Nadeln: $\nu=1906,1926,2015 \mathrm{~cm}^{-1}$ (CO). MS [m/z (rel. Int. \%)]: $M^{+} 742(2.6), M^{+}-\mathrm{CO} 714(0.1), M^{+}-2 \mathrm{CO} 686$ (3.9), $M^{+}-3 \mathrm{CO} 658$ (8.3), $M^{+}-4 \mathrm{CO} 630$ (22.6), $M^{+}-5 \mathrm{CO} 602$ (39.7), $M^{+}-5 \mathrm{CO}-\mathrm{Me}$ 587 (13.7), $M^{+}-6 \mathrm{CO} 574(8.5), M^{+}-6 \mathrm{CO}-\mathrm{Me} 559$ (65.5), $M^{+}-6 \mathrm{CO}-2 \mathrm{Me}$ 
544 (5.0), $\mathrm{CMe}_{3}^{+} 57$ (70.8), $\mathrm{CO}^{+} 28$ (100.0). Gef. C, 51.55; H, 5.89. $\mathrm{C}_{34} \mathrm{H}_{46} \mathrm{Mo}_{2} \mathrm{O}_{6}$ (742.6) Ber. C, 54.99; H 6.24\%.

(Die Abweichung im Kohlenstoff-Wert ist auf die Bildung von Molybdäncarbid (MoC) zurückzuführen).

Dank. Wir danken der Deutschen Forschungsgemeinschaft und dem Fonds der Chemischen Industrie für die gewährte finanzielle Unterstützung.

\section{Literatur und Bemerkungen}

1 P. Jutzi, J. Schnittger und M.B. Hursthouse, Chem. Ber., im Druck.

2 M.D. Rausch, W.C. Spink, B.G. Conway, R.D. Rogers und J.L. Atwood, J. Organomet. Chem., 383 (1990) 227.

3 M.J. Freeman, A.G. Orpen, N.G. Connelly, I. Manners und S.J. Raven, J. Chem. Soc., Dalton Trans., (1985) 2283.

4 M. Tilset und K.P.C. Vollhardt, Organometallics, 4 (1985) 2230.

5 R. Brand, H.-P. Krimmer, H.-J. Lindner, V. Sturm und K. Hafner, Tetrahedron Lett., 23 (1982) 5131.

6 W.P. Fehlhammer, W.A. Herrmann und K. Öfele, in G. Brauer (Hrsg.), Handbuch der Prăparativen Anorganischen Chemie, III. Aufl., Ferdinand-Enke-Verlag. Stuttgart, 1981, III. Bd., S. 1914.

7 D.W. Macomber und M.D. Rausch, J. Organomet. Chem., 258 (1983) 331.

8 J.C. Smart und C.J. Curtis, Inorg. Chem., 16 (1977) 1788.

9 Daten zur Strukturanalyse von 3 (Standardabweichungen sind in Klammern angegeben). Summenformel $\mathrm{C}_{34} \mathrm{H}_{46} \mathrm{Mo}_{2} \mathrm{O}_{6}$; Molmasse $742.6 \mathrm{~g} / \mathrm{mol}$; Gitterkonstanten $a=25.339$ (5) $\dot{A}, b=8.9370$ (12) $\dot{A}$, $c=18.603(5) \AA, \beta=123.41(1)^{\circ} ;$ Zellvolumen $3516.6(12) \AA^{3}, Z=4 ; d_{\text {ber }}=1.403 \mathrm{~g} / \mathrm{cm}^{3}$; Linearer Absorptionskoeffizient $0.733 \mathrm{~mm}^{-1} ; F(000)=1528$; Kristallsystem monoklin, Raumgruppe $C 2 / c$; Meßgerăt: Vierkreisdiffraktometer Siemens P2 ${ }_{1}$; Strahlung Mo-K $K_{\alpha} \lambda=0.71073 \AA$; Meßtemperatur $20^{\circ} \mathrm{C}$; Meßbereich $4 \leqslant 2 \theta \leqslant 50^{\circ} ; h, k, l$ Grenzen $0 \rightarrow-30,-10 \rightarrow 0,-18 \rightarrow 22$; Absorptionskorrektur semi-empirisch; Zahl der gemessenen Reflexe 3486, davon unabhängige 2217 mit $I \geqslant 2 \sigma(I)$; Strukturaufklärung mit direkten Methoden; full matrix Verfeinerung, alle Nicht-Wasserstoff-Atome anisotrop, Wasserstoffatome an berechneten Positionen mit fixem $U=0.08 \AA^{2}$; Anzahl der verfeinerten Parameter 190; Reflex : Parameter-Verhălnis 11.7:1; $R=3.63 \% ; R w=3.72 \%$ mit $w^{-1}=\sigma^{2}\left(F_{0}\right)$. Weitere Einzelheiten zur Kristallstruktur von 3 können beim Fachinformationszentrum Karlsruhe, Gesellschaft für wissenschaftlich-technische Information mbH, W-7514 Eggenstein-Leopoldshafen 2, unter Angabe der Hinterlegungsnummer CSD-55358, der Autorennamen und des Zeitschriftenzitats angefordert werden.

10 W.A. Herrmann, D. Andrejewski und E. Herdtweck, J. Organomet. Chem., 319 (1987) 183.

11 M.R. Churchill und J. Wormald, Inorg. Chem., 8 (1969) 1970.

12 M.A. Greaney, J.S. Merola und T.R. Halbert, Organometallics, 4 (1985) 2057.

13 J.S. Drage und K.P.C. Vollhardt, Organometallics, 5 (1986) 280. 